\title{
Aerodynamic Characteristic Analysis of Multi-Rotors Using a Modified Free-Wake Method
}

\author{
By Jaewon LEE, ${ }^{1)}$ Kwanjung $\mathrm{YEE}^{2)}$ and Sejong $\mathrm{OH}^{2)}$ \\ ${ }^{1)}$ Research Institute of Mechanical Technology, Pusan National University, Busan, Korea \\ ${ }^{2)}$ Department of Aerospace Engineering, Pusan National University, Busan, Korea
}

(Received November 23rd, 2008)

\begin{abstract}
Much research is in progress to develop a next-generation rotor system for various aircrafts, including unmanned aerial vehicles (UAV) with multi-rotor systems, such as coaxial and tandem rotors. Development and design of such systems requires accurate estimation of rotor performance. The most serious problem encountered during analysis is wake prediction, because wake-wake and wake-rotor interactions make the problem very complex. This study analyzes the aerodynamic characteristics based on the free-wake method, which is both efficient and effective for predicting wake. This code is modified to include the effect of complex planforms as well as thickness by using an unsteady 3D panel method. A time-marching free-wake model is implemented based on the source-doublet panel method that assigns panels to the surface and analyzes them. The numerical wake instability, the most critical problem for analysis, is resolved by adopting slow start-up and by including viscous effects. Also, the instability due to wake interference in tandem rotor analysis is resolved by configuring the initial shapes of the multi-rotor wake as that of a single rotor wake trajectory. The developed code is verified by comparing with previous experimental data for coaxial and tandem rotors.
\end{abstract}

Key Words: Multi Rotor, Time-Marching Free-Wake, Unsteady Panel Method, Wake Instability

\section{Nomenclature}

$c$ : blade chord

$C_{d}$ : rotor overlap area

$C_{\mathrm{p}}$ : pressure coefficient

$C_{\mathrm{p}_{0}}$ : incompressible pressure coefficient

$C_{\mathrm{P}}$ : rotor power coefficient

$C_{\mathrm{T}}$ : rotor thrust coefficient

$d$ : horizontal distance between rotors

$D$ : diameter of rotor disk

$H$ : vertical separation between rotors

$M$ : mach number

$r_{\mathrm{c}}$ : vortex core radius

$R$ : radius of blade

Re: Reynolds number

$t$ : maximum airfoil thickness

$v_{\text {rel }}:$ relative velocity

$\bar{V}$ : velocity

$\Gamma$ : circulation

$\kappa$ : induced power factor

$\mu$ : doublet

$\theta$ : collective pitch angle

$\sigma:$ source

$\sigma_{\text {rotor }}:$ rotor solidity

$\Phi$ : velocity potential

$\psi$ : azimuth angle

$\psi_{\text {wake: }}$ wake age

\section{Introduction}

An anti-torque system is redundant in a multi-rotor helicopter, such as one with tandem or coaxial rotors. The substantial advantage of a multi-rotor system stems from the elimination of the tail rotor, which usually consumes an additional $5 \%$ to $10 \%$ of the main rotor's power. On the other hand, the mechanical complexity and associated parasitic drag are believed to be major disadvantages, which have made this configuration relatively unpopular so far. However, the absence of a tail rotor allows lowers the number of helicopter accidents associated with the tail rotor, and enables a more compact configuration than conventional helicopters. Hence, due to its compact size and lift ability, the multi-rotor system is seem as a viable alternative in UAV or micro-air vehicle (MAV) design. ${ }^{1)}$ In response to increasing needs for compact multi-purpose UAVs, the dual rotor system has been chosen in this work. As shown in Coleman's comprehensive review ${ }^{2)}$ of experimental and analytical studies of coaxial rotors, studies during the 1990s relied heavily on experiments, with a few theoretical analyses. Moreover, there is still a long unresolved issue about whether or not the coaxial rotor configuration is more efficient than the conventional rotor system. Detailed aerodynamic characteristics, such as wake structures and interrotor interactions, have never been fully understood for coaxial rotor configurations.

However, recent advances in computational techniques have enabled modeling of such complex aerodynamics with good confidence. Leishman and Ananthan ${ }^{3)}$ established the Blade Element Momentum Theory (BEMT) for a coaxial 
rotor and solved the local air loads over the upper and lower rotors. The BEMT does not require the assumptions for the induced power factor $(\kappa)$ and induced power interference factor $\left(\kappa_{\mathrm{int}}\right)$, which are essential for the simple momentum theory. Validation of BEMT with Harrington's experiments ${ }^{4)}$ has shown good agreement. With the limited test data available, Leishman and Ananthan ${ }^{3)}$ have compared the spanwise airloads of BEMT for a coaxial rotor with the results of a Free-Vortex Method (FVM). ${ }^{5)}$ Although differences were evident when compared in detail, the agreement between BEMT and FVM was found to be reasonable. Based on 2D theory, BEMT has the remarkable advantage of very low computational cost, while it has limitations in analyzing flight regimes such as forward flight, for which 3D effects are important. One research that used full 3D theory, was by Bagai and Leishman ${ }^{5)}$ for analysis of multi-rotors using the potential theory-based vortex wake method with pseudo-implicit predictor-corrector method. Another study by Wachspress and Quackenbush ${ }^{6)}$ used the constant vorticity contour method. Although these studies have proved successful and computationally efficient, the methods use a vortex lattice description of the blade and cannot predict thickness effects. Moreover, complex configurations of blades with variations in the sectional airfoil have rarely been considered. Due to the relative motion of the rotors, multi-rotor analysis using Navier-Stokes (NS) based methods requires a complicated grid topology and involves significant difficulties in pre- and post-processing. One instance of a multi-rotor study using a NS-based method was by Kim and Brown, ${ }^{7)}$ who numerically analyzed the performance of a coaxial rotor in hover, steady forward, and level flight conditions, using a Vorticity Transport Model (VTM) based on a time-dependent computational solution of the vorticity conservation form of the NS equation. The simulation results provided a detailed analysis of the differences in performance and the wake behavior of the single and coaxial rotor systems. Another application of an NS-based method to a coaxial rotor was by Ruzicka and Strawn. ${ }^{8)}$ They described efforts at modeling coaxial rotor aerodynamics using the Reynolds-averaged, NavierStokes (RANS) solver Overflow 2. The VTM, which is formulated assuming incompressibility, is a partial CFD scheme with NS for the near blade region, but RANS is a fully viscous CFD scheme. When using a convection algorithm and an adaptive-grid system, VTM was more efficient than regular CFD analysis using the NS equation. However, it is still computationally inefficient compared to potential theory based panel methods.

Vortex wake methods represent less diffusive numerical schemes that accurately reproduce the wake and are more efficient than NS codes. They are still used widely for helicopter aerodynamic analysis. However, as mentioned previously for the Vortex Lattice Methods (VLM), which represent a blade as vortex lattices, the existing vortex wake methods also have limitations in accounting for rotor complexity, including thickness variation and airfoil change by location, such as found in UH-60 rotor blades. This research intends to develop a code for analysis of a multi-rotor UAV system based on the vortex wake method. The developed method favors analysis of complex wakes generated by rotors, and also includes the effects of rotor thickness. The current study considers an unsteady source-doublet panel method coupled with a time-marching free-wake model. In the single-rotor case, the concept of a finite-thicknessbladed rotor was presented by Ahmed and Vidjaja, ${ }^{9)}$ who used a source/sink distribution over the blade surface and a doublet distribution over the camber surface inside the blade. However this study used source and doublet distributions over the blade surface. To improve the calculation efficiency for wake-induced velocity, the doublet panel with constant strength representing the wake is replaced by an equivalent vortex trapezoid. An important issue regarding codes using a time-marching free-wake model is resolution of wake instability in hover. This paper focuses on resolving this, issue and the interaction of rotor wakes. The KarmanTsien compressibility correction is applied in order to correct the compressibility effect over the calculated pressure coefficient. The Table-lookup method, which is usually used supplementary in codes that do not account for viscous effects, is used for estimating power of the rotor system. For UAVs or model rotors, due to the difference in the Reynolds numbers between the analysis and the tables for 2D airfoil aerodynamic data, the Reynolds number scaling law is used to estimate rotor power.

Validation of results for multi-rotor configurations from developed code has been carried out by comparison with available experimental data. ${ }^{4,10}$ The trajectory of the tip wake in the present method is compared with the experimental data of Nagashima, ${ }^{11)}$ and Brown. ${ }^{7)}$ The effects of the tandem rotor vertical separation $(H / D)$ and horizontal distance $(d / D)$ are investigated thoroughly.

\section{Numerical Analysis}

To account for the effect of thickness and the complex platform, this work uses a source-doublet panel method, which is usually used in fuselage or full aircraft analysis of fixed-wing aircraft. In addition, this work uses a timemarching free-wake model, which is excellent in reproducing rotor wake. The problem in applying the time-marching free-wake model is wake instability. The following sections describe the solution to this problem, as well as the numerical method used in the multi rotor analysis code.

\subsection{Source-doublet panel method}

If flow is considered to be incompressible and irrotational, the continuity equation reduces to the Laplace equation. The general solution of the Laplace equation is given as a sum of sources $(\sigma)$ and doublets $(\mu)$, distributed over the body surface and its wakes:

$$
\begin{aligned}
\Phi= & \frac{-1}{4 \pi} \int_{\text {body }}\left[\sigma\left(\frac{1}{r}\right)-\mu \hat{n} \cdot \nabla\left(\frac{1}{r}\right)\right] \mathrm{d} s \\
& +\frac{1}{4 \pi} \int_{\text {wake }}\left[\mu \hat{n} \cdot \nabla\left(\frac{1}{r}\right)\right] \mathrm{d} s+\Phi_{\infty}
\end{aligned}
$$


However, Eq. (1) still does not describe the solution uniquely, because a large number of sources and doublets are required to satisfy the set of boundary conditions. To determine the unique solution, the source distribution is set to be equal to the local kinematic velocity. To establish the boundary value problem, the local velocity at each collocation point on the body must satisfy the zero-flow condition across the body surface:

$$
\nabla \Phi \cdot \hat{n}-\vec{V}_{\infty} \cdot \hat{n}=0
$$

Also, from the Kutta condition, the wake doublets can be expressed in terms of the unknown surface doublets. When specified at the body's $N$ collocation points, Eq. (2) takes the form:

$$
\left[A_{i j}\right]\left(\mu_{k}\right)=\left(R H S_{k}\right)=\left[B_{i j}\right]\left(\sigma_{k}\right)+\left[C_{i j}\right]\left(\mu_{w k}\right)
$$

This matrix has a nonzero diagonal and a stable numerical solution. The resulting pressures can be computed using the Bernoulli equation, yielding:

$C_{\mathrm{p}}=-\frac{(\nabla \Phi)^{2}}{v_{\mathrm{ref}^{2}}}+\frac{2}{v_{\mathrm{ref}^{2}}}\left[\vec{V}_{0}+\vec{\Omega} \times \vec{r}\right] \cdot \nabla \Phi-\frac{2}{v_{\mathrm{ref}^{2}}} \frac{\partial \Phi}{\partial t}$

Furthermore, the Karman-Tsien compressibility correction, shown in Eq. (5), is applied to the pressure coefficient. Although the Prandtl-Glauert correction is simplest, it tends to underestimate the pressure coefficient when compared to empirical data, while correction with Karman-Tsien's, which accounts for nonlinear aspects of flow, gives more accurate results. ${ }^{12)}$

$$
C_{\mathrm{p}}=\frac{C_{\mathrm{p}_{0}}}{\sqrt{1-M_{\infty}^{2}}+\left(\frac{M_{\infty}{ }^{2}}{1+\sqrt{1-M_{\infty}^{2}}}\right) \frac{C_{\mathrm{p}_{0}}}{2}}
$$

Figure 1 compares the distribution over the NACA 0012 airfoil calculated using the 2D source-doublet panel code with compressibility correction and a experiment conducted by Harris, ${ }^{13)}$ for Mach number 0.551 and angle of attack of 5 degrees. Karman-Tsien's correction law appears to best agree with the experiment results.

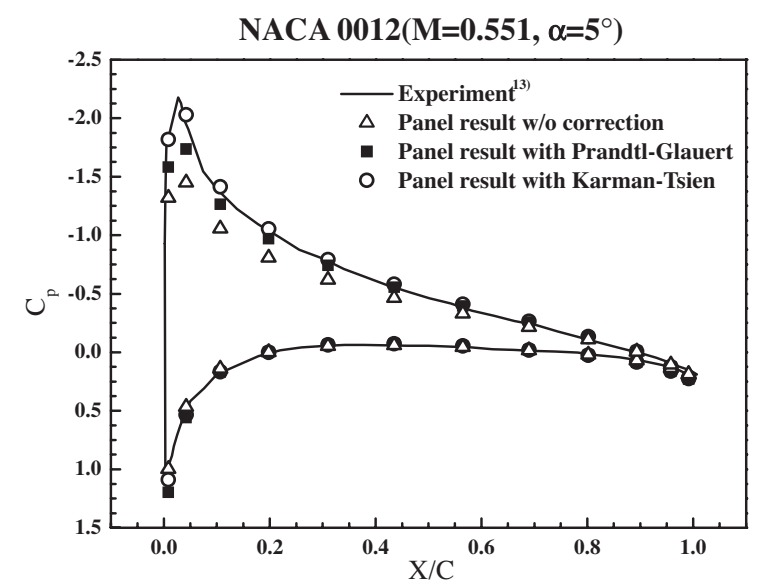

Fig. 1. Comparison of several compressibility corrections for NACA 0012 airfoil.

\subsection{Time-marching free-wake model}

A wake model is needed to simulate wake deformation caused by contraction and displacement. The free-wake concept allows for vorticity evolving in a free motion, and represents the physically correct approach of unsteady aerodynamics. The model includes a wake-relaxation scheme and a time-marching scheme. The time-marching free-wake model is used in this work. The wake is shed from the trailing edge of the rotor, and its size increases linearly with the time step. For a fixed number of time steps, the time-marching method is roughly twice as fast as the wake-relaxation scheme. ${ }^{14)}$

The wake-doublet panel of constant strength is replaced by an equivalent vortex trapezoid because it is more efficient to calculate induced velocity using the Biot-Savart law. This approach is based on Hess ${ }^{15)}$ who showed that a constantstrength doublet element is equivalent to a constant strength vortex trapezoid placed at the panel edges. By using a vortex trapezoid instead of a doublet element, it is possible to use both a vortex-core model and a vortex-core growing model. The total induced velocity at a point is obtained by summation of the velocity induced by each vortex segment. The induced velocity is given by the Biot-Savart law for a potential vortex as:

$$
\vec{V}=\frac{\Gamma}{4 \pi} \int_{1} \frac{\vec{d} l \times \vec{r}}{|\vec{r}|^{3}}
$$

As found from Eq. (6), the velocity field has a singularity at the vortex center. This singularity problem is resolved by using a vortex core model. Leishman, et al. have suggested that the Vatistas vortex model, ${ }^{16)}$ with $n=2$, would fit the experimental data best. ${ }^{17}$ The present method uses the Vatistas vortex model to prevent this singularity. With this model, the equation for the induced velocity is modified to:

$$
\vec{V}_{\mathrm{p}}=\frac{\Gamma}{2 \pi} \frac{h}{\left(r_{\mathrm{c}}^{2 n}+h^{2 n}\right)^{1 / n}}\left(\cos \theta_{1}-\cos \theta_{2}\right) \vec{e}
$$

where, $n$ is an integer (Fig. 2).

Applying the time-marching free-wake model with the source-doublet panel method may cause part of the wake panel to appear inside the blade surface when the blade intersects the wake. In such a case, the wake panel inside the blade would violate the solid boundary condition. It is physically impossible for non-zero flow across a solid

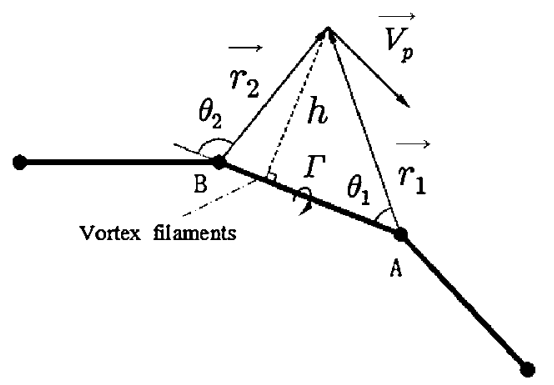

Fig. 2. Schematic showing velocity induced by straight-line vortex element. 
surface. Hence, a reasonable solution cannot be obtained using Eq. (3) derived from the solid boundary condition. In reality, as vortex filaments reach the solid surface they are distorted to form loop or hairpin vortex structures and bursts. ${ }^{18)}$ Such problems also occur in VLM for analyses that include fuselages. To resolve the issue, several ideas have been tried. Mavris et al. ${ }^{19)}$ simply ignored the vortex filaments inside the airframe volume when computing the induced velocity. Quackenbush et al. ${ }^{20)}$ developed a "fat core" model, based on the classical panel method to prevent development of singularities when the vortex approaches too close to the surface panel points. Crouse ${ }^{21)}$ used a source panel description of the fuselage - a lifting line blade model and a free tip vortex wake model, without an inboard wake model-to represent the rotor/fuselage system. Vortex wake elements that cross the fuselage surface were handled by splitting them into smaller segments, and shifting the collocation points of these smaller segments so that they were at a specified minimum distance from the fuselage surface. Since the collision of the wake and the blade cannot be taken into account in the fat core model, it prevents interaction of the blade and wake that may occur in forward flight. Crouse's method increases the number of wake elements for the frequent collision of the blade and the wake, causing increased computing time. Therefore the problem was resolved by eliminating the potential value of the wake vortex trapezoid inside the solid boundary. The basic concept of the present method is similar to that used by Mavris et al. ${ }^{19)}$ vortex trapezoids meeting the solid surface are eliminated completely and are not used in calculation. This concept was first suggested by Lighthill. ${ }^{22)}$

\subsection{Wake instability}

Instability of the rotor wake is an important issue when analyzing hover performance using the free-wake model. During analysis, instabilities in the wake arise due to interactions between the strong starting vortex, created by the impulsive starting motion, and the wake created at the rotor hub. This is a general problem that occurs in hovering rotor analyses with a free-wake based panel method.

There have been various attempts to overcome this problem. Chung and Lee ${ }^{23)}$ used slow start up giving a downward induced velocity to the wake for up to 2 revolutions. Rosen et al. ${ }^{24)}$ assumed that the vortex filament at the hub travels at a speed that is $75 \%$ of that at the tip. Summa and Maskew ${ }^{25}$ ) calculated the free-wake with an advance ratio of 0.05 and then corrected the calculation for the hovering condition with test data. Bagai and Leishman ${ }^{26)}$ stated that an artificial large vortex core radius is necessary to avoid abrupt changes in the angle of attack due to large induced velocities created between adjacent vortices and the tip vortex very close to the blade. Furthermore, Quaranta et al. ${ }^{27)}$ rapidly increased the core radius of the starting vortex and the inner vortex to achieve a stable and accurate solution. When applied to VLM without accounting for thickness, most methods used by researchers so far have little effect, if any, on the sourcedoublet panel method that accounts for thickness. In addition to the methods mentioned, other attempts have been

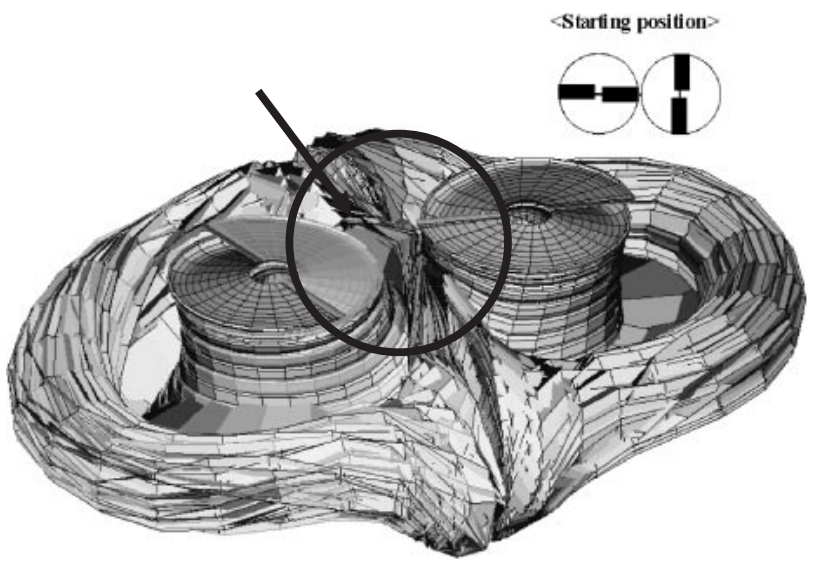

(a) Wake instability factor (20 revolutions)

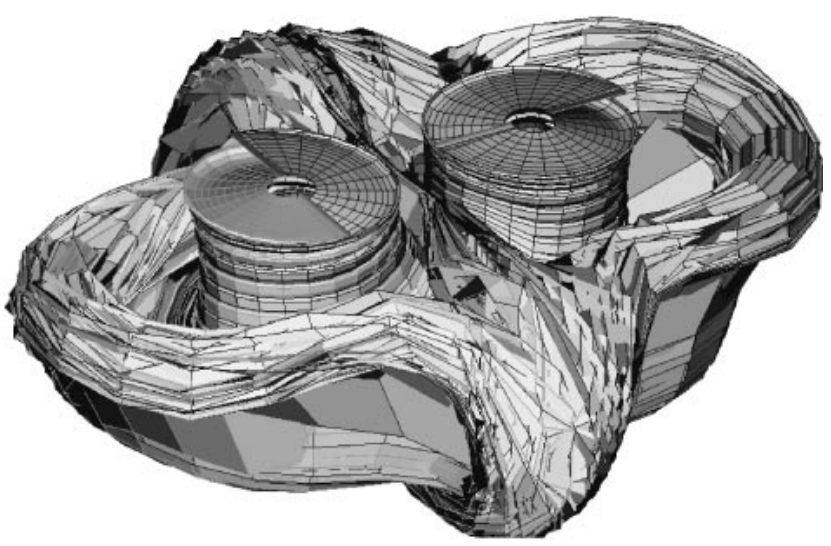

(b) Wake instability (27 revolutions)

Fig. 3. Wake instability due to wake-wake interaction.

tried without noticeable effects, including prescribing a climb speed that is $5 \%$ of the tip speed. This is followed by hovering.

This work achieves a stable and accurate solution by applying slow start up for the first two revolutions to reduce the effects of the starting vortex and the viscous effects that Leishman et al. ${ }^{28)}$ suggested. The vortex core diffusion effects are modeled using the following empirical relation:

$$
r_{\mathrm{c}}\left(\psi_{\text {wake }}\right)=\sqrt{r_{\mathrm{c} 0}^{2}+4 \alpha \delta v \psi_{\text {wake }} / \Omega}
$$

Where, $r_{\mathrm{c} 0}$ is the initial core size, $\alpha$ is the Lamb-Oseen coefficient (1.25643), $\delta$ is the average effective viscosity coefficient, and $v$ is the kinematic viscosity. The initial core size is used as $10 \%$ of the chord length that is commonly used in rotor wake simulations. Representative values of $\delta$ for helicopter rotor vortices range from 10 (at model scale) up to more than 1000 (at full scale). ${ }^{28)}$

As another illustration of the wake instability problem, Fig. 3 shows that a larger inflow is calculated at the border region of the rotors without any treatment, and results in overestimation of the overlap induced interference power factor $\left(\kappa_{\mathrm{ov}}\right)$ for a tandem rotor configuration, with the distance between the rotors, $d / D$, of 1.25 for the same rotor height. This occurs because the wakes created from the rotors during the initial calculations stay right under the 


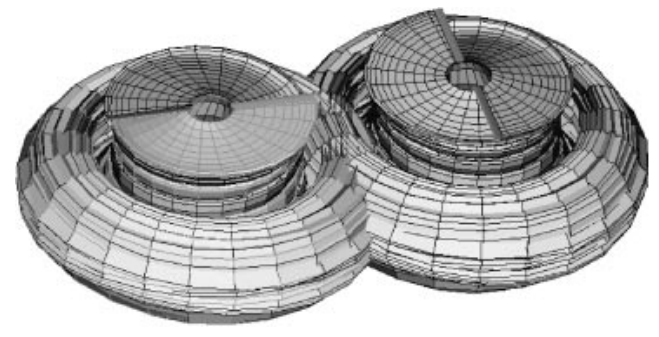

(a) Initial wake geometry (wake age $=13$ revolutions)

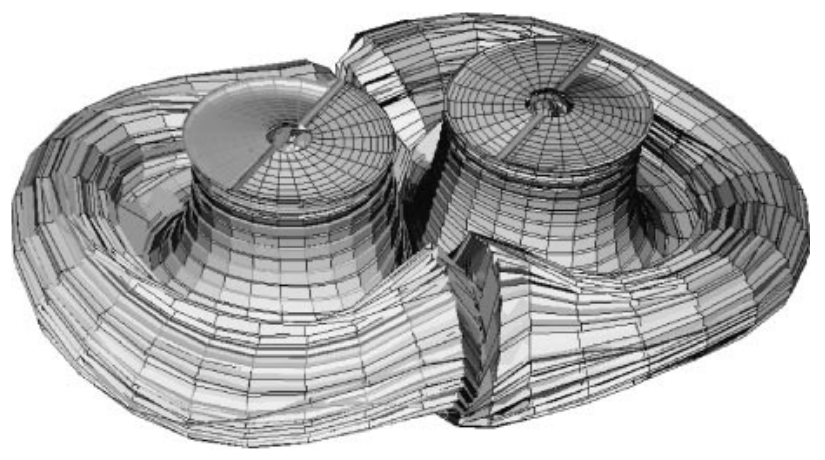

(b) Wake geometry after 7 rotations (wake age $=20$ revolutions)

Fig. 4. Wake instability treatments using single rotor wake geometry.

rotor disks, affecting each other. The strong vortex near the rotor disk causes a large inflow on the rotor, and hence obstructs accurate wake predictions. This phenomenon is seldom found physically, because in general, rotorcrafts hover after take-off and climb. Therefore, the interference effects of the strong wake created initially are relatively small. In addition, similar wake trajectories are evident only in cases $^{29)}$ with ground effects, while the tip wake easily proceeds downward in cases without the ground effect, so the wake instability problem due to wake-wake interactions in the present method must be resolved.

To resolve instabilities caused by the stationary wake near the rotor disk, the initial wake trajectory at single rotor analysis is imposed for the wake geometry of each rotor as shown in Fig. 4(a) and then analysis proceeds for the tandem rotor configuration. Although fluctuations appear at the start of calculation, they converge after five revolutions. Figure 4(b) shows the disappearance of the lump of the wake under the rotor disk, removing the instabilities in the wake. Applying the wake trajectory for a single rotor starts the calculations with the lump of the wake away from the rotor disk, preventing interference between rotor wakes at the start. A similar approach has been used elsewhere. ${ }^{29)}$ In addition, replacing the initial revolution with that for a single rotor significantly reduces the total calculation cost.

\section{Result and Discussion}

\subsection{Hovering coaxial rotor system}

For coaxial rotor systems, the results of the present code were compared with the experimental data by Harrington. ${ }^{4)}$ Two coaxial rotor configurations were tested in the Harring-

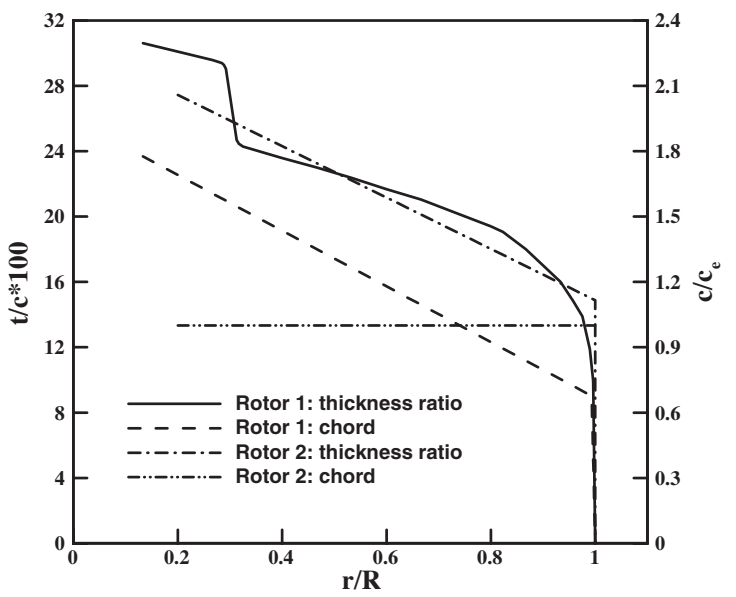

Fig. 5. Variation of rotor blade chord and thickness ratio of Harrington's rotors.

ton's experiment where the coaxial rotors consisted of two counter-rotating, two-blade teetering rotors 7.62 meters in diameter. One rotor system ( $1 \mathrm{st}$ case) had a solidity, $\sigma_{\text {rotor }}$, of 0.027 , with two blades per rotor $\left(2 \sigma_{\text {rotor }}=0.054\right.$ for coaxial rotor) with $H / D=0.19 R$. The other rotor system (2nd case) also had two blades per rotor with a solidity of $0.076\left(2 \sigma_{\text {rotor }}=0.152\right.$ for the coaxial rotor) with $H / D=$ $0.16 R$. Both sets were untwisted but the 1 st case had a tapered blade. The equivalent blade chord for the 1 st case is $0.1616 \mathrm{~m}$ and the blade chord for the 2nd case is $0.4572 \mathrm{~m}$. Both rotor systems had variable thicknesses along their spans. The aerodynamic characteristics of most airfoil sections are unavailable although the blade sections were based on the NACA 4-digit series. Details of the planform and the thickness ratio of the blades are shown in Fig. 5.

Since the source-doublet panel codes cannot consider viscous drag, the table for the two-dimensional airfoil aerodynamic performance must be provided to correctly calculate the profile rotor power. With Harrington's rotors having airfoil sections with varying thickness ratio, it is necessary to use proper airfoil aerodynamic tables for look-up to estimate power according to the thickness of the airfoil. However, generating the airfoil data through wind tunnel tests, or CFD, takes time. In this work, for efficient calculation, the power required was estimated using corrected drag coefficients, accounting for the thickness based on the NACA0012 airfoil table obtained from tests. The aerodynamic drag of the non-standard airfoil sections were obtained from the empirical correlation suggested by Leishman: ${ }^{18)}$

$$
C_{d_{0}} \approx 0.007+0.025\left(\frac{t}{c}\right)
$$

Airfoil tables are usually given for high Reynolds number ranges $\left(10^{6}-10^{7}\right)$. It was necessary to scale the given airfoil table to match the current Reynolds number because the drag-and eventually the profile rotor power-is very sensitive to the Reynolds number. Wachspress and Quackenbush $^{6)}$ suggested that it would be sufficient to assume that: 


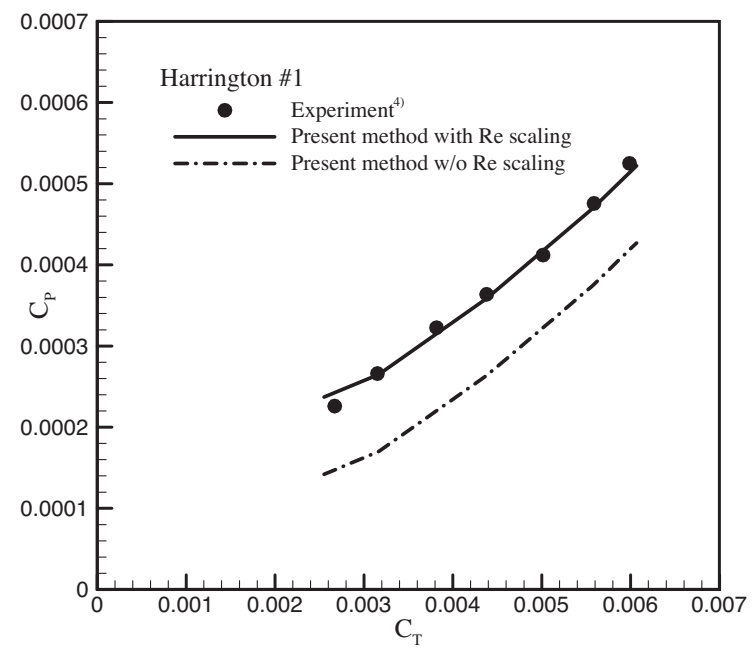

(a)

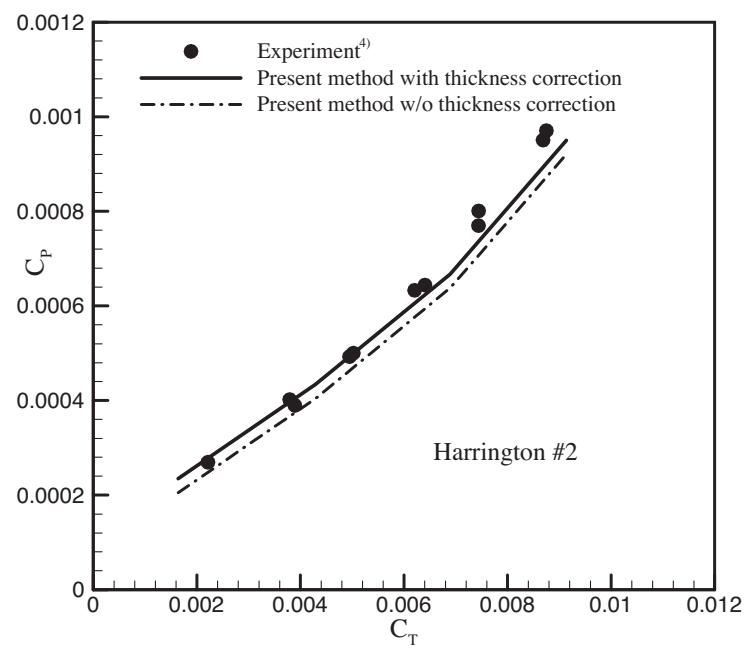

(b)

Fig. 6. $C_{\mathrm{T}}$ vs. $C_{\mathrm{P}}$ for Harrington's rotor (a) 1 st case and (b) 2 nd case.

$$
C_{d_{\text {min,sub }}}=C_{d_{\text {min,full }}}+C_{d_{\text {min,full }}}\left(\operatorname{Re}_{\text {full }} / \operatorname{Re}_{\text {sub }}\right)^{1 / 5}
$$

as long as the practical range of the full scaled rotor's Reynolds number was above $10^{6}$. It is known that Reynolds number scaling is inadequate at very low Reynolds numbers, below the $3 \times 10^{5}$ to $5 \times 10^{5}$ range. The Reynolds number was corrected using Eq. (10) in this work.

Figure 6 shows the performance of the coaxial rotors at hover, revealing that the analysis results for the complex configuration agree very well with the experimental data for the tip Mach number of 0.293 . The Reynolds numbers based on the rotor tip speed for Harrington's rotor systems 1 and 2 were $8 \times 10^{5}$ and $2.3 \times 10^{6}$, respectively. The reference Reynolds number from the aerodynamic data, given in C81 format, was $5.2 \times 10^{6}$ and the drag coefficients were corrected based on the local Reynolds number using Eq. (10). The differences in the thrust and power with respect to the drag correction due to the Reynolds number are shown in Fig. 6(a), indicating that the accuracy of the Reynolds number significantly impacts the accuracy of the performance analysis. Figure 6(b) compares the results with

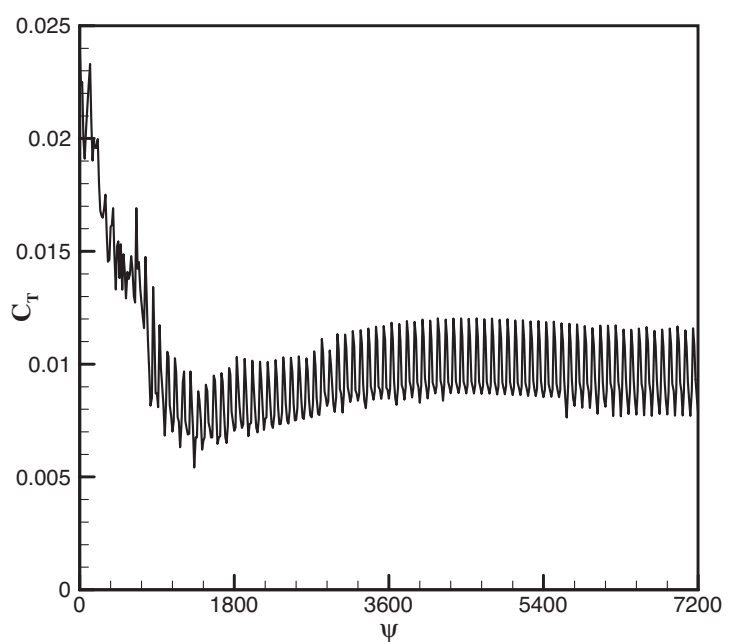

Fig. 7. Thrust coefficient histories of Nagashima's coaxial rotor.

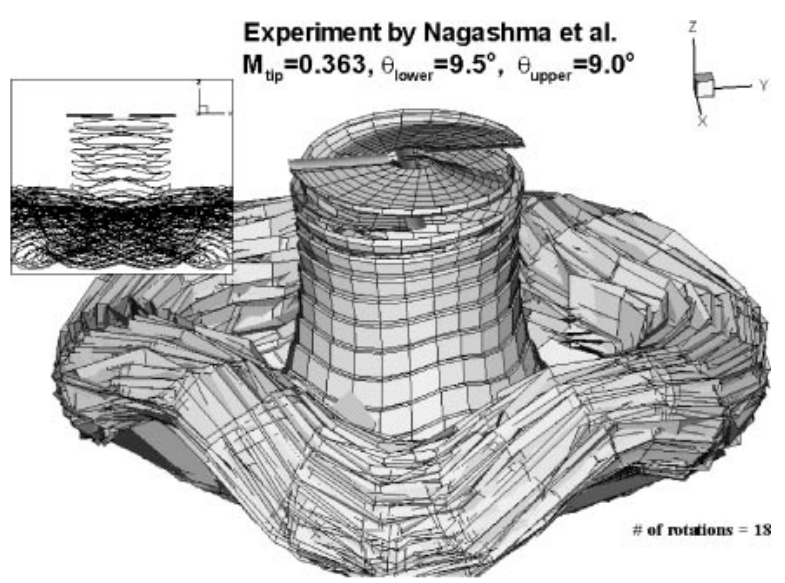

Fig. 8. Wake geometries of Nagashima's coaxial rotor at 18 revolutions.

the thickness-effect-corrected drag coefficients and with a uniform NACA0012 airfoil, regardless of the thickness ratio in the experiment data. The results accounting for the thickness effect follow the experimental data better than those without thickness correction.

For a detailed verification of the contraction and the axial descent rate of the tip vortices generated by the coaxial rotor at hover, the present calculations were compared with the experimental data by Nagashima et al. ${ }^{11)}$ The coaxial rotor used in the experiment consisted of two articulated rotors of rectangular planform with NACA 0012 airfoil sections and a blade chord of $0.06 \mathrm{~m}$. The rotor blades were untwisted with a diameter of $0.76 \mathrm{~m}$. The rotor tip Mach number was 0.363 . The experimental data shown in Fig. 7 were obtained for a vertical separation of $H / D=0.105$. In the experiment, the collective pitches of the upper and lower rotors were fixed at $9^{\circ}$ and $10^{\circ}$, respectively, achieving a torque balance. In this study, following Kim and Brown's strategy, ${ }^{7)}$ the calculated results were obtained by trimming each rotor to match the experimental thrust $\left(C_{\mathrm{T} \text {,upper }}=0.0059, C_{\mathrm{T}, \text { lower }}=\right.$ 0.0038), rather than merely setting the rotors to the same collective pitch as in the experiment. The collective pitches of the upper and lower rotors were replaced with $9^{\circ}$ and $9.5^{\circ}$ 

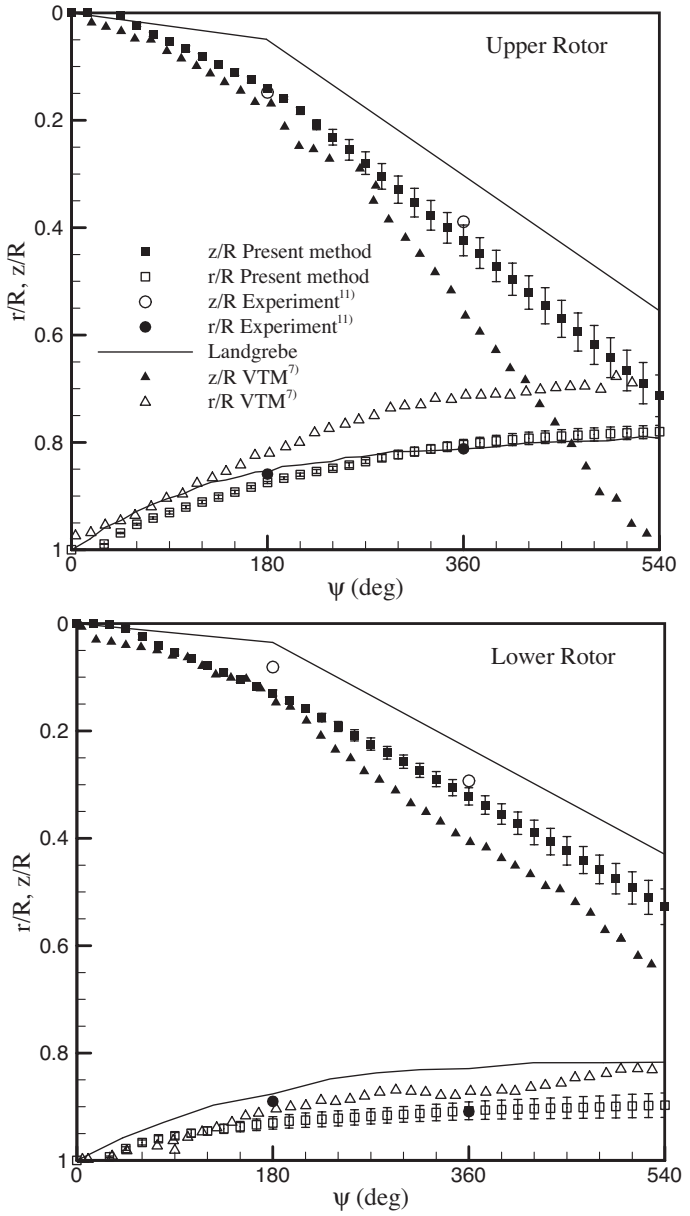

Fig. 9. Correlation of tip vortex trajectory of coaxial rotor.

in the calculation. $\mathrm{C}_{\mathrm{T}}$ converged after 16 revolutions. Figure 7 and 8 show the convergence history and wake geometry at revolution 18 .

As seen in Fig. 9, the overall agreement between the calculations and experiments is good, although the numbers in the experimental data are in sufficient for rigorous comparison. The tip vortex trajectory obtained here was averaged over one revolution. The error bars in the figure indicate the range of location variation. The calculations replicate the physical trends observed by previous researchers; the axial descent rate of the tip vortices of both rotors is faster than that of the individual rotors. However, the computations seem to show a slower radial contraction of the tip vortices on the lower rotor than for the same rotor in isolation.

Figure 10 show the tip vortex trajectories at two representative azimuth angles. The results show that the tip vortex trajectories of both rotors oscillate along the azimuth angle. This is attributed to the varying relative position of the upper and lower rotor blades due to the counter rotation. From Fig. 10(a), the tip vortex generated by the upper rotor blade 1 passes the lower rotor plane at around $220^{\circ}$ and meets another tip vortex generated by the lower rotor at $225^{\circ}$. On the other hand, Fig. 10(b) shows that they intersect at an angle of $260^{\circ}$. After that, oscillations occur at the tip vortex trajectory and amplitude grows, as shown in Fig. 9. These oscillations depend on the relative positions of the

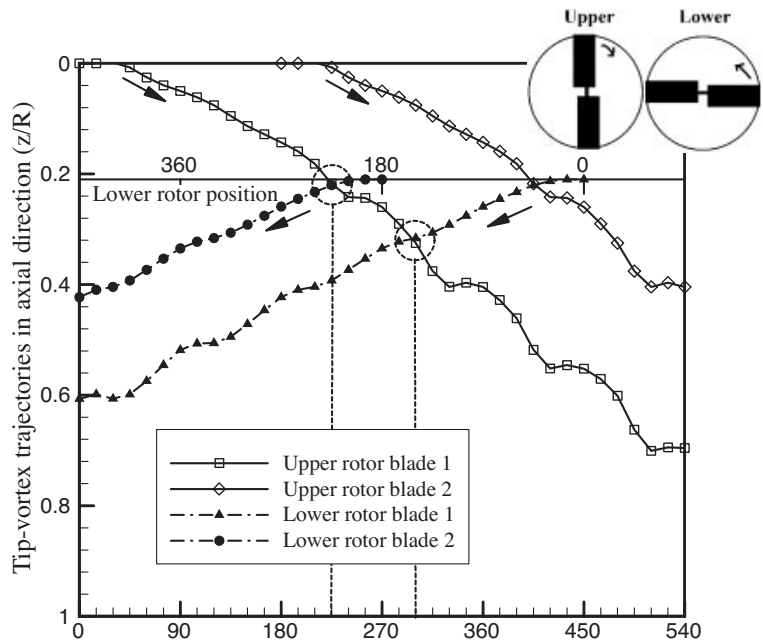

(a)

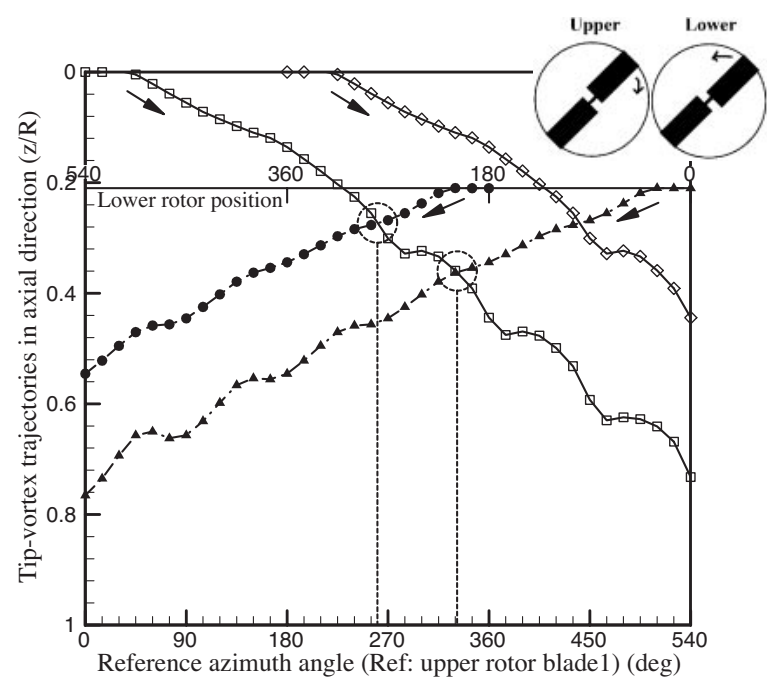

(b)

Fig. 10. Tip vortex trajectory of coaxial rotor in axial direction.

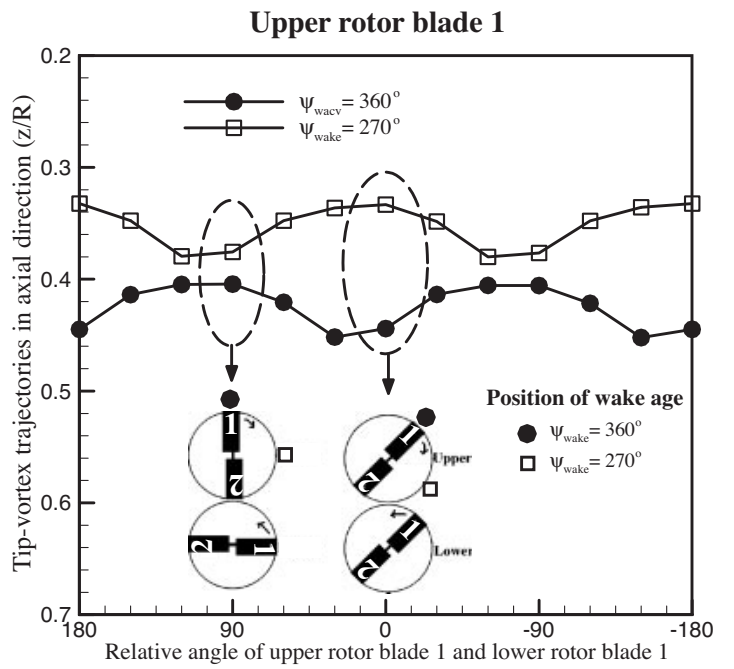

Fig. 11. Vortex oscillation trajectory of upper rotor blade 1 . 


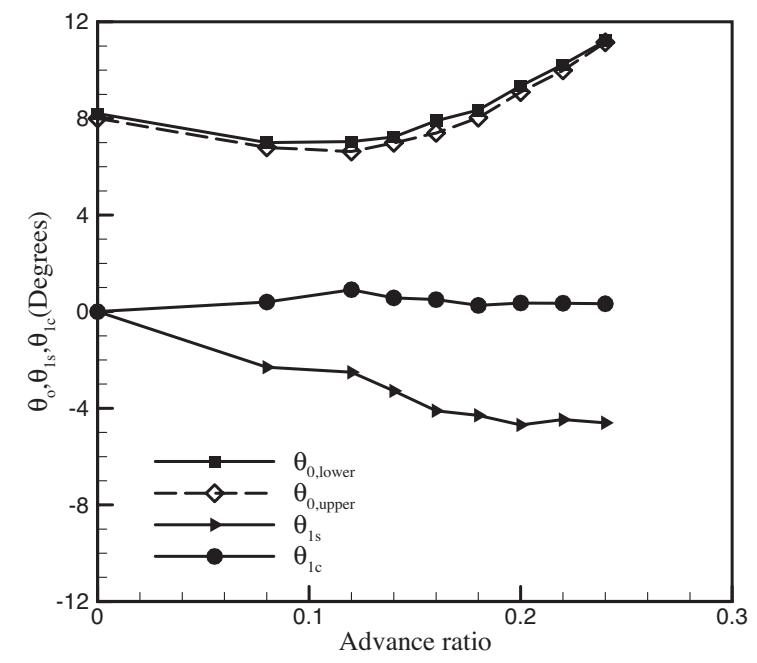

Fig. 12. Control angles required for trim in forward flight.

wake and rotor blade as shown in Fig. 11, illustrating the changes in the tip vortex at two points with different wake ages with respect to the relative angles between the upper rotor blade 1 and the lower rotor blade 1 . When there is a $90^{\circ}$ phase difference, the lower rotor blade 1 is located over the $270^{\circ}$ wake-aged tip vortex of the upper rotor blade 1 , whereas it is over the $360^{\circ}$ wake-aged vortex in the absence of a phase difference. Comparing the trajectories on the graph shows that both wakes drop in the axial direction approaching the lower rotor blade 1 and vice versa. This shows that oscillations of the tip vortex trajectories depend on the relative positions of the upper and lower rotors. Due to the lack of sufficient test data, the actual occurrences of such tip vortex trajectories, which are not mentioned clearly in Brown's work, were difficult to verify. Hence, further research is needed.

\subsection{Forward flight case of coaxial rotor system}

The present calculations of the coaxial rotor performance in forward flight are compared with the wind tunnel data from Dingeldein's investigations. ${ }^{10)}$ The rotor used in this study was identical to Harrington's rotor 1 . The experimental trim strategy of using a differential collective pitch to trim the rotor to zero net yawing moment was replicated in the calculations. To represent the effects of the fuselage parasitic drag on the trim state of the experimental system, the forward tilt of the rotor was adjusted to produce a sufficient forward force to overcome the drag corresponding to a fuselage equivalent flat plate area of 0.93 square meters, which was used in the experiment. The trim calculation was automated using a Newton-Raphson iterative method. Figure 12 shows the control inputs required to trim the rotor. These include the collective pitch $\left(\theta_{0}\right)$, the longitudinal cyclic $\left(\theta_{1 \mathrm{~s}}\right)$ and the lateral cyclic $\left(\theta_{1 \mathrm{c}}\right)$. The upper and lower rotors in these simulations were given equal cyclic pitch inputs. The trim converged after 3-4 iterations. The initial values affect the convergence rate. Figure 13 shows the comparison between the present simulation, VTM simulation $^{7)}$ and Dingeldein's measurements ${ }^{10)}$ of power consumption against the advance ratio for a uniform thrust coefficient

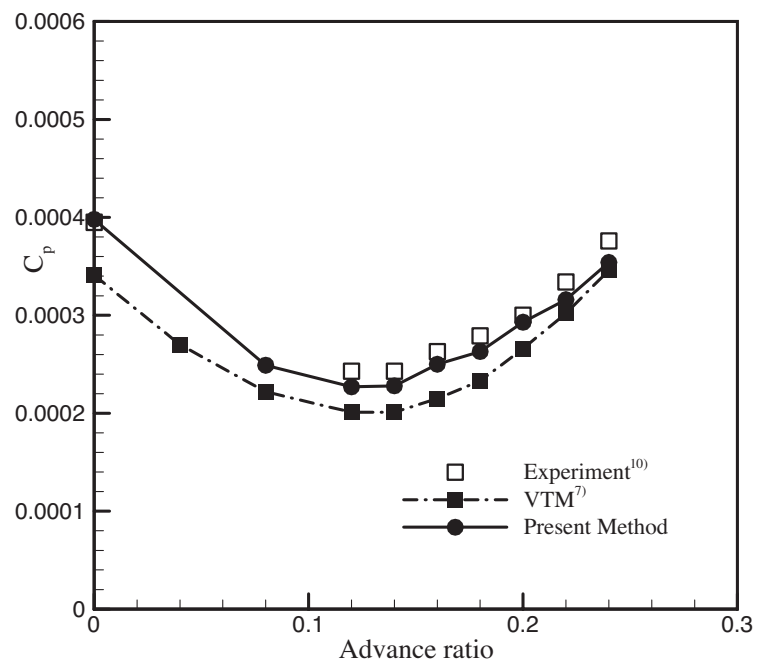

Fig. 13. Overall power consumption in steady forward flight $\left(C_{\mathrm{T}}=0.0048\right)$.

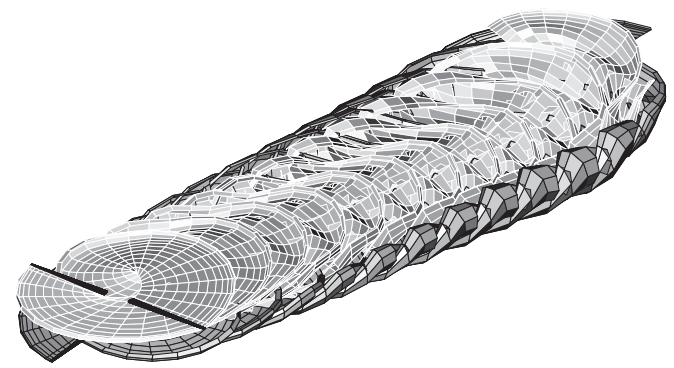

Fig. 14. Wake geometry of coaxial rotor in steady forward flight (advanced ratio $=0.20)$.

of 0.0048 with a rotor tip Mach number of 0.448 . It shows that the experimental power trend for the coaxial rotor is well captured by the present method. The tread of the present calculations coincides with these experiments, and the present estimates are more accurate than the VTM results, which systematically underestimate the power coefficient at all advance ratios. Kim and Brown argued that this power consumption was due to uncertainties in the profile drag characteristics of the rotor. Although both, the VTM and present method use the same table look-up method to estimate the profile power, the method in this study appears to reproduce the test data with better agreement due to its better estimation of the wake trajectory, as shown in Fig. 9. The VTM method results in a faster downward movement of the tip vortex than the test data. This seems to be due to a larger impact of the wake on the blades caused by overestimation of induced velocity, leading to underestimation of profile power. On the other hand, the present approach models the behavior of the wake very well, resulting in better estimation of the profile power. The wake geometry of coaxial rotors in forward flight is shown in Fig. 14, indicating that the wake of lower rotor rolls up and wraps the wake of the upper rotor.

\subsection{Hovering case of tandem rotor system}

The experimental data of Dingeldein ${ }^{10)}$ was considered to validate the tandem rotor hover case. Dingeldein's tandem 


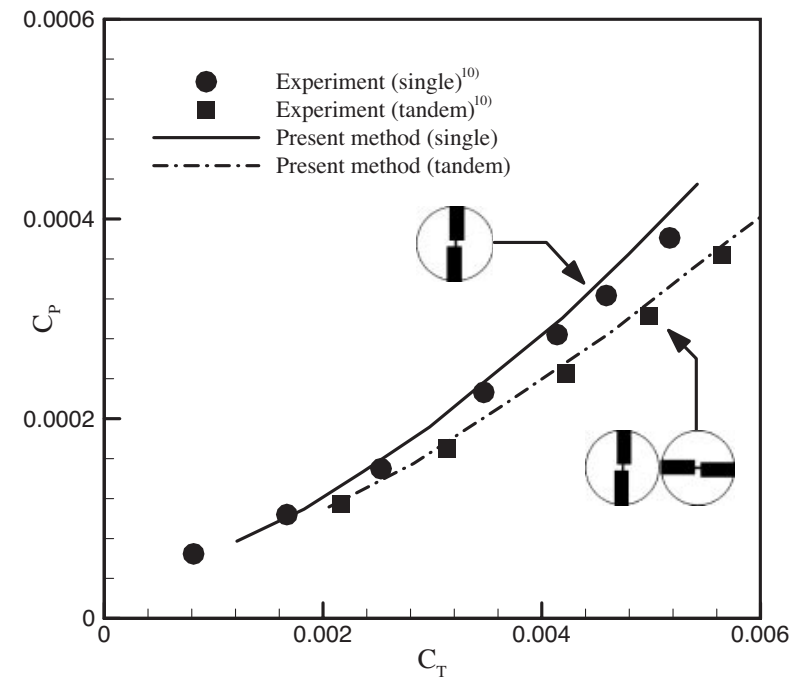

Fig. 15. Comparison of experiment and numerical result (tandem rotor).

model consisted of a two-blade rotor, $4.57 \mathrm{~m}$ in diameter. The rotor shafts were parallel, with $d / D=1.03$. Each rotor had a solidity of 0.054 . The blades were untwisted and untapered and had a NACA 0012 airfoil section with a chord of $0.194 \mathrm{~m}$. Figure 15 shows the comparison between the current simulations of the rotor power versus thrust and Dingeldein's data, for both tandem and isolated rotors. The tip Mach number of the rotor blades is 0.448 . The computed results are in good agreement with the measurements in both cases. The power coefficient matched better at low thrust coefficients than at higher values, which is attributed to calculating the required power using a table look-up procedure. To calculate the rotor profile power using panel methods, which do not account for viscous effects, the drag coefficient was found from the 2D airfoil table, considering the Mach number and the lift coefficient calculated by the present method at a particular rotor blade section. The profile power is obtained by integrating the $2 \mathrm{D}$ drag coefficient at each section along the blade. Considering the relation between lift and drag coefficients, it is well known that the variation of drag coefficient becomes larger at large lift coefficient. In other words, drag estimates based on table look-ups would be more accurate at low lift coefficients. Therefore, as shown in Fig. 15, the results agree better at lower thrust regimes both for single or tandem rotors. In addition, comparing the single rotor to the tandem rotor shows that, for the same $C_{\mathrm{T}}$, the error in the power estimation of the single rotor is greater than that of the tandem rotor. For the same $C_{\mathrm{T}}$, the single rotor must generate twice the thrust as the tandem configuration. Therefore, the $C_{1}$ calculated for the single rotor blade section must be greater than for the tandem rotor, resulting in larger errors in the profile power estimation as shown in Fig. 15.

Since experimental data validating wake prediction of a tandem rotor in hover are hard to find, the performance of the tandem rotor was compared indirectly with results on the overlapping effects of a tandem rotor due to wake interference. The overlapping effects were calculated at various

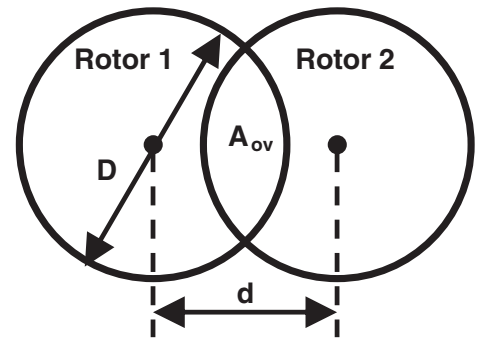

Fig. 16. Definition of overlap (plan).

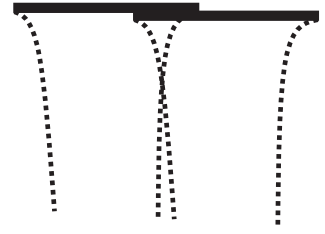

Case 1

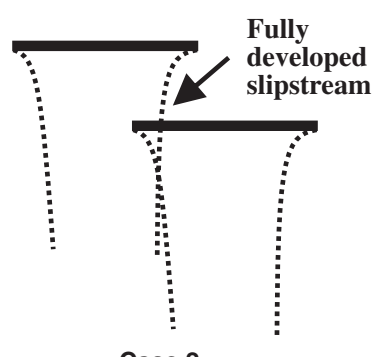

Case 2
Fig. 17. Flow models used for tandem rotor analysis.

spacing intervals, $d$, between the two rotor axes for a tandem rotor system with the same diameter, $D$. In addition, the effect of the vertical interval, $H$, was analyzed. The overlapping effect was investigated by comparing the overlap induced interference power factor, $\kappa_{\mathrm{ov}}$, which is the total induced power of the overlapped rotors divided by the total induced power of two isolated rotors. Prior research has extended the momentum theory to calculate the overlapping effects of the tandem rotor, stating that it depends on the overlap area and the vertical interval $(H / D)$ of the rotors. The definition of overlap area is shown in Fig. 16. As the effect of wake changes with the vertical interval $(H / D)$ of the rotors, two assumptions (Fig. 17), are made. First, the rotors are assumed to have no vertical spacing (Case 1). The second assumption is to consider a situation where the lower rotor in tandem operates in the fully contracted slipstream of the upper rotor (Case 2 ). In case $1, \kappa_{\mathrm{ov}}$ appears to be the ratio of the total induced power of the two isolated rotors and the total induced power of overlapped rotors. In case 2 , the lower rotor is affected by the upper rotor after the wake is contracted. Therefore, the induced velocity should be found by numerical iteration.

In this work, Dingeldein's tandem rotor, ${ }^{10)}$ which was previously used for the hovering performance validation, was selected to investigate the overlapping effects for various $d / D$ and $H / D$ values. Results from momentum theory were compared with the present calculation (Fig. 18). The calculations tend to be similar to the momentum theory. However, the values predicted by the present method are smaller than the results of momentum theory, in general. This occurs because closer spacing of the rotors causes less contraction of wakes created at each rotor due to the interference between them. The tip vortex of 5 recent revolutions is shown in Fig. 19 for better clarity. A similar 


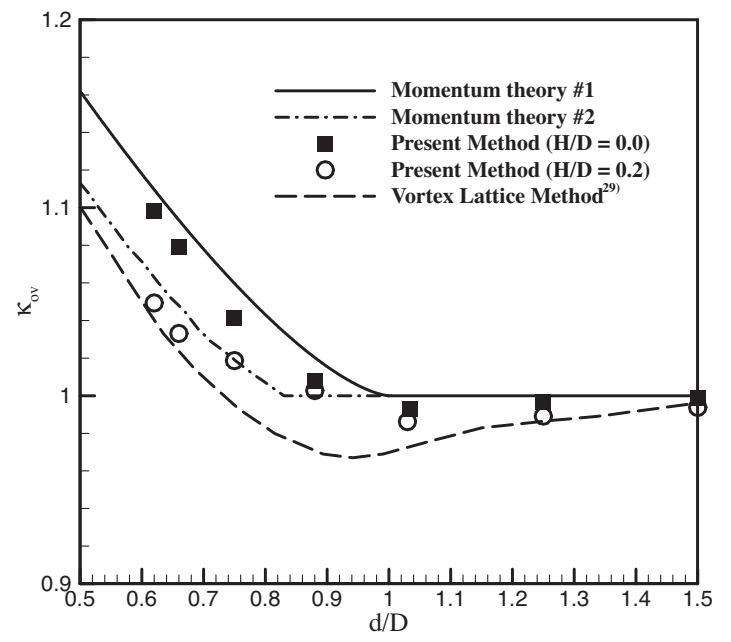

Fig. 18. Overlap induced interference factor vs. rotor separation distance.

trend was found in a previous study. ${ }^{29)}$ Less contraction of the rotor wake makes the area of the vena contracta relatively larger, causing reduced speeds in that region. The continuity equation for hover flight, ${ }^{18)}$ states that the reduced speed due to the increased area of vena contracta is followed by a decrease in the induced speed at the rotor disk, and hence reduced induced power.

Figure 18 shows a very similar trend to the momentum theory for $d / D$ less than 0.9 . The case with $H / D$ set to 0.0 is equivalent to case 1 of the momentum theory, while the case with $H / D$ set to 0.2 is equivalent to case 2 , in which the wake generated at the upper rotor contracted sufficiently. At the region where $d / D$ is greater than 0.9 , the present code and results from previous research ${ }^{29)}$ show some part where $\kappa_{\mathrm{ov}}$ is less than 1.0. The case 2 model of the momentum theory states that $\kappa_{\mathrm{ov}}$ is 1.0 , because the fully contracted wake does not pass through the lower rotor disk. The reason is that the present code accounts for the effects of interaction between the wakes generated at each rotor, while the momentum theory does not. As shown in Fig. 20(a), the momentum theory does not alter the inflow speed, depicted by the downward arrows if there is no overlap. However, it is known that the induced velocity generated from one rotor wake causes an upward velocity on the other rotor near the border, and hence reduces the average wake speed, followed by a lesser induced velocity at each rotor. $^{28)}$ Figure 20(b) shows the inflow speed-decrease mechanism caused by wake interaction. It also shows that the region with $\kappa_{\mathrm{ov}}$ below 1.0 exists because of the decrease in induced power due to the interactions. As shown in Fig. 18, a similar region where $\kappa_{\mathrm{ov}}$ is less than 1.0 appears in this study as well. The benefit of the overlap effect due to a $\kappa_{\mathrm{ov}}$ value of less than 1.0 on a tandem rotor has been proved experimentally. ${ }^{10)}$ However, the previous results ${ }^{29)}$ show a greater drop in $\kappa_{\mathrm{ov}}$, resulting in differences compared to this work. According to Ref. 30), the downwash due to the generated wake varies with the rotor configuration, altering the growth or contraction of the other rotor wake. Therefore, there is a difference in the induced veloc-

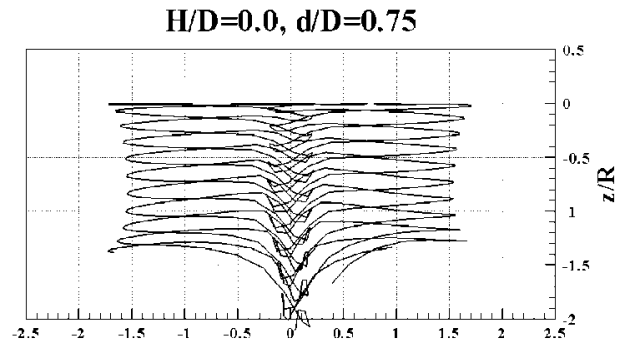

$\mathbf{s} / \mathbf{R}$

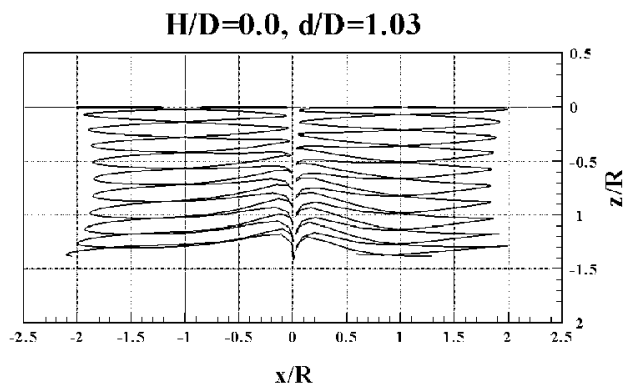

$\mathrm{H} / \mathrm{D}=\mathbf{0 . 0}, \mathrm{d} / \mathrm{D}=\mathbf{1 . 2 5}$

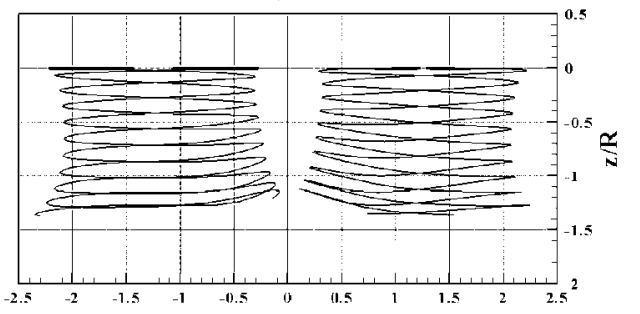

$\mathrm{x} / \mathrm{R}$

$\mathrm{H} / \mathrm{D}=\mathbf{0 . 2}, \mathrm{d} / \mathrm{D}=\mathbf{0 . 7 5}$

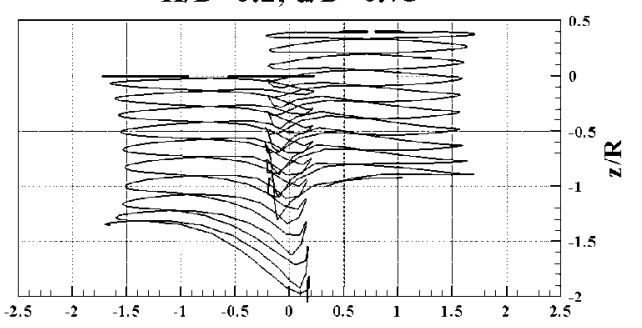

$\mathrm{x} / \mathrm{R}$

$H / D=0.2, d / D=1.03$

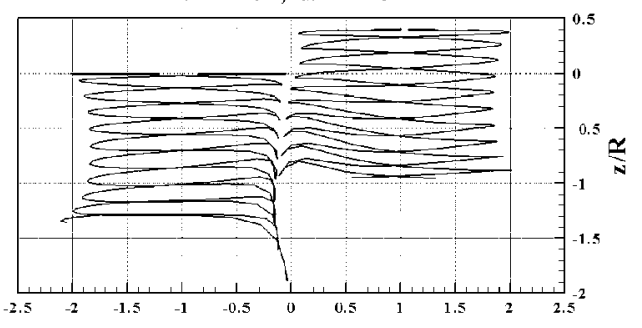

$\mathrm{x} / \mathrm{R}$

$H / D=0.2, d / D=1.25$

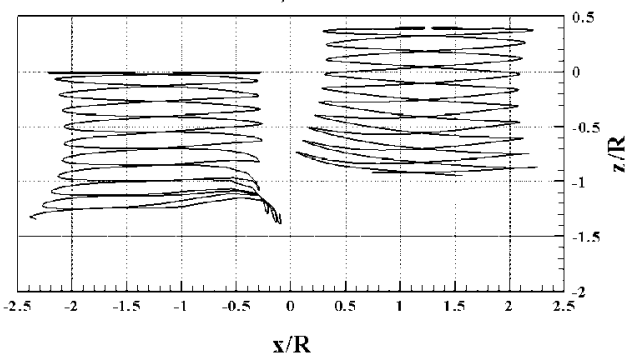

Fig. 19. Wake predictions for hovering tandem rotor staggers. 


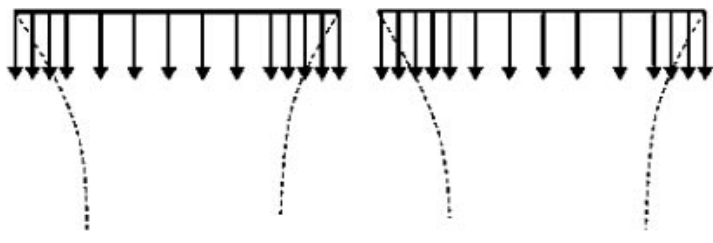

(a) Without wake interference effect

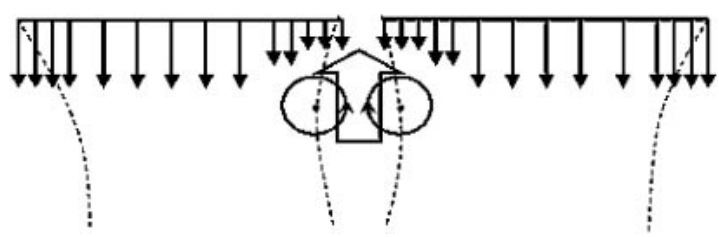

(b) With wake interference effect

Fig. 20. Schematics of wake interference effect.

ity depending on the severity of the interaction. The differences in the results of this analysis and Ref. 29) also appear to be due to different rotor configurations. The three-bladed rotor used in the previous analysis ${ }^{29)}$ is for a CH-46 and 47, with V23010-1.58 airfoil, $-10^{\circ}$ twist and a $20 \%$ root cut. On the other hand, this work uses a twobladed rotor with NACA0012 airfoil, no twist, no taper and a rectangular tip.

\section{Conclusions}

To investigate the aerodynamic characteristics of a multirotor system, an analysis code was developed based on the free-wake method. The code can analyze the complex wake geometry formed by the interaction between rotors, and including the thickness effects of rotors, which the existing free-wake methods cannot handle. To estimate power, table look-up was used in the unsteady panel code, coupled with a time-marching free-wake model. The wake instability caused by wake-wake interaction in the specified rotor distance was treated by using single-rotor wake geometry as the initial shape of the multi-rotor wake. Also, Reynolds number scaling was used for cases of different Reynolds numbers. The developed method was validated by comparing the numerical results for the multi-rotor configurations with experimental data. The computed results showed good agreement with the experimental data. The following conclusions have been reached:

(1) To account for thickness effects, a code incorporating a time-marching free-wake model was constructed based on a source-doublet panel method. The code was validated by various experiment cases, showing very accurate prediction of aerodynamic performance.

(2) The most challenging problem during development was wake instability. Instabilities due to impulsive starting were resolved by applying slow start up and by including viscous effects. Instabilities due to interaction between multi-rotor wakes were resolved by presetting the initial wake trajectory with the one found in single-rotor analysis.
(3) The tip vortex trajectories of the coaxial rotor showed very good agreement with the experimental data. The trajectory appeared to oscillate according to the relative locations of the upper and lower rotors. The trajectory drops down and goes up in the axial direction when the tip vortex location, in terms of wake age, approaches and leaves the rotor blade, respectively. It has been shown that prediction of accurate wake trajectory by the current code provides a generally accurate induced velocity as well as estimate of rotor performance.

(4) The results for the overlapping effects of tandem rotor appeared to be similar to the momentum theory. In addition, a region of induced interference with beneficial effects was found near $d / D=1$.

\section{Acknowledgments}

This study was supported by the KARI under KHP Dual-Use Component Development Program funded by the Ministry of Knowledge Economy.

\section{References}

1) Bohorquez, F., Samuel, P., Sirohi, J., Pines, D. and Rudd, L.: Design, Analysis and Performance of a Rotary Wing MAV, J. Am. Helicopter Soc., 48 (2003), pp. 80-90.

2) Coleman, C. P.: A Survey of Theoretical and Experimental Coaxial Rotor Aerodynamic Research, NASA Technical Paper 3675, 1997.

3) Leishman, J. G. and Ananthan, S.: Aerodynamic Optimization of a Coaxial Proprotor, The American Helicopter Society 62nd Annual Forum, Phoenix, USA, 2006.

4) Harrington, R. D.: Full Scale Tunnel Investigation of the Static Thrust Performance of a Coaxial Helicopter Rotor, NACA TN 2318, 1951.

5) Bagi, A. and Leishman, J. G.: Free-wake Analysis of Tandem, Tilt-Rotor and Coaxial Rotor Configurations, J. Am. Helicopter Soc., 41 (1996), pp. 196-207.

6) Wachspress, D. A. and Quackenbush, T. R.: Impact of Rotor Design on Coaxial Rotor Performance, Wake Geometry and Noise, The American Helicopter Society 62nd Annual Forum, Phoenix, USA, 2006.

7) Kim, H. W. and Brown, R. E.: Coaxial Rotor Performance and Wake Dynamics in Steady and Maneuvering Flight, The American Helicopter Society 62nd Annual Forum, Phoenix, USA, 2006.

8) Ruzicka, G. C. and Strawn, R. C.: Computational Fluid Dynamics Analysis of a Coaxial Rotor Using Overset Grids, The AHS Specialist's Conference on Aeromechanics, San Francisco, USA, 2008.

9) Ahmed, S. R. and Vidjaja, V. T.: Unsteady Panel Method Calculation of Pressure Distribution on BO 105 Model Rotor Blades, J. Am. Helicopter Soc., 43 (1998), pp. 47-56.

10) Dingeldein, R. C.: Wind-Tunnel Studies of the Performance of Multi Rotor Configurations, NACA TN 3236, 1954.

11) Nagashima, T. and Nakanishi, K.: Optimum Performance and Wake Geometry of Coaxial Rotor in Hover, Proc. of the 7th European Rotorcraft and Powered Lift Forum, Paper No. 41, 1981.

12) Anderson, J. D.: Modern Compressible Flow with Historical Perspective, 3rd ed., McGraw-Hill, Singapore, 2003.

13) Harris, C. D.: Two-dimensional Aerodynamic Characteristics of the NACA 0012 Airfoil in the Langley 8-Feet Transonic Pressure Tunnel, NACA TM 81927, 1981.

14) Katz, J. and Plotkin, A.: Low-Speed Aerodynamics, 2nd ed., Cambridge University Press, Cambridge, 2001.

15) Hess, J. L. and Smith, A. M. O.: Calculation of Potential Flow about Arbitrary Bodies, Progress in Aeronautical Sciences, Pergamon Press, New York, 1967. 
16) Vatistas, G. H., Kozel, V. and Mih, W.: A Simpler Model for Concentrated Vortices, Exp. Fluids, 11 (1991), pp. 73-76.

17) Leishman, J. G., Baker, A. and Coyne, A.: Measurements of Rotor Tip Vortices Using Three-Component Laser Doppler Velocimetry, J. Am. Helicopter Soc., 41 (1996), pp. 342-353.

18) Leishman, J. G.: Principles of Helicopter Aerodynamics, 2nd ed., Cambridge University Press, New York, 2006.

19) Mavris, D. N., Liou, S. G., Komerath, N. M. and McMahon, H. M.: Measurement and Computation of the Velocity Field of a Cylinder in the Wake of a Rotor in Forward Flight, Paper AIAA-89-1844, The 20th AIAA Fluid Dynamics, Plasma Dynamics, and Lasers Conference, Buffalo, NY, June 12-14, 1989.

20) Quackenbush, T. R., Lam, C. G. and Bliss, D. B.: Vortex Methods for the Computational Analysis of Rotor/Body Interaction, J. Am. Helicopter Soc., 39 (1994), pp. 14-24.

21) Crouse, G. L., Jr.: An Analytical Study of Unsteady Rotor/Fuselage Interaction in Hover and Forward Flight, $\mathrm{PhD}$ Thesis, University of Maryland, USA, 1992.

22) Lighthill, M. J.: Laminar Boundary Layers, Dover Publications, Inc., Oxford, 1963.

23) Chung, K. H. and Lee, D. J.: Numerical Prediction of Rotor TipVortex. Pairing by Using a Time-Marching Free-Wake Method, Comput. Fluid Dynam. J., 12 (2003), pp. 80-88.

24) Rosen, A. and Graber, A.: Free Wake Model of Hovering Rotors
Having Straight or Curved Blades, J. Am. Helicopter Soc., 33 (1988), pp. 11-19.

25) Summa, J. M. and Maskew, B.: New Method for the Calculation of Hover Air Loads, Paper No. 15, Proc. of the Fifth European Rotorcraft and Powered Lift Aircraft Forum, Amsterdam, The Netherlands, 1979.

26) Bagai, A. and Leishman, J. G.: Rotor Free-Wake Modeling Using a Relaxation Technique-Including Comparisons with Experimental Data, The 50th Annual Forum of the American Helicopter Society, Washington, D.C., USA, May 11-13, 1994.

27) Quaranta, G., Bindolino, G., Masarati, P. and Mantegazza, P.: Toward a Computational Framework for Rotorcraft Multi-Physics Analysis: Adding Computational Aerodynamics to Multibody Rotor Models, The 30th European Rotorcraft Forum, Marseilles, France, Sep. 1416, 2004.

28) Leishman, J. G., Bhagwat, M. J. and Bagai, A.: Free-Vortex Filament Methods for the Analysis of Helicopter Rotor Wakes, J. Aircraft, 39 (2002), pp. 759-775.

29) Griffiths, D. A. and Leishman, J. G.: A Study of Dual-Rotor Interference and Ground Effect Using a Free-Vortex Wake Model, American Helicopter Society 58th Annual Forum, Montreal, Canada, June 1113, 2002.

30) Stepniewski, W. Z. and Keys, C. N.: Rotary-Wing Aerodynamic, Dover, Canada, 1984. 